\title{
Arbor
}

\section{Introducción: Hacia una «cultura de los Servicios de Inteligencia» ${ }^{1}$}

\author{
Fernando García Sanz
}

Arbor CLXXX, 709 (Enero 2005), 1-21 pp.

"A los españoles nos repugna sobremanera la palabra espionaje. Nuestro temperamento no está formado para emplearnos en tan bajos menesteres. El espionaje, como otros vicios que se extienden por España, ha sido importado del otro lado de los Pirineos, y es una planta exótica en nuestro país. Por eso los españoles no sabremos ser jamás espías de ninguna nación, ni incluso de la nuestra. En cambio, el español habilidoso, audaz y travieso, riéndose de esta clase de maniobras, sin espiar a nadie, sabrá explotar la candidez de los extranjeros que creen sin duda que eso del espionaje sirve para algo más que para sostener a cuatro vivos".

En estos términos resumía en su editorial del 30 de junio de 1918 el periódico La Tribuna de Madrid su opinión sobre una actividad, la del espionaje, sobre la que se llevaba hablando en España prácticamente desde el inicio de la Gran Guerra, alimentando crónicas en la prensa generalmente inducidas por las propias redes de espionaje y contraespionaje de los beligerantes ${ }^{2}$. El momento era muy significativo. Hacía pocos días

\footnotetext{
1 El presente trabajo se inscribe en el marco del proyecto de investigación (McyT, BHA2002-01143) Espionaje y relaciones internacionales: los servicios de información aliados en España durante la Primera Guerra Mundial.

2 El título completo del largo editorial de La Tribuna era «En plena orgía financiera. Los grandes negocios del espionaje aliadófilo. Romanones, Urgoiti y el órgano de La Papeleran.
} 
que el Comisario de policía destinado en Barcelona, Manuel Bravo Portillo, junto a sus principales ayudantes, había dado con sus huesos en la cárcel acusado, precisamente, de formar parte de la red de espionaje alemana con sede en la capital de Cataluña. Fue la prensa, apoyada por los servicios de información de la Entente, la que denunció al personaje y a sus más inmediatos colaboradores, sacando a la luz una extensa trama y aportando para ello pruebas que resultaban incontestables. El texto completo del editorial con el que se abren éstas líneas, venía a refutar la tesis de la existencia misma de la trama y a considerar, aún con mayor fuerza, que ciudadanos españoles pudieran estar implicados en ella ${ }^{3}$. En todo caso, el tema respondería -obediencia financiera obliga- a un montaje de los enemigos de Alemania y quedaba, como toda responsabilidad de Bravo y compañeros, el haber sabido sacar partido -comportamiento pícaro, este sí muy español-a unas circunstancias -las impuestas por el espionaje internacional- de las que en absoluto se les podía considerar responsables. ¿Por qué los españoles que se daban como implicados, a pesar de las pruebas, no podían tener ninguna relación con el escándalo? La respuesta que da el periódico entra dentro de los márgenes del más arraigado casticismo: Porque el español estaría adornado de una serie de virtudes («la hidalguía y la caballerosidad, rasgos fulminantes del carácter español», se diría en otro lugar) incompatibles con el ejercicio del espionaje, al que se le otorgan toda una serie de connotaciones peyorativas que se sabe ancladas en el imaginario colectivo, entre las cuales el permanente engaño, la traición y la corrupción serían sus principales señas de identidad ${ }^{4}$. En conclusión, ajenos pues los españoles «por idiosincra-

${ }^{3}$ LA TRIBUNA, diario de Madrid, había sido creado en 1913 por el polémico diputado y periodista S. Cánovas Cervantes. De línea liberal-independiente pasó pronto a convertirse en el órgano de prensa maurista y una vez que estalló la guerra se fue inclinando progresivamente hacia el bando alemán hasta que, después de la Batalla del Marne, pasó a ser subvencionado por los alemanes que, primero, compraban millares de ejemplares y después llegaron a instalar una nueva rotativa, pagar el telégrafo y aportar 15.000 pesetas todos los meses. De sus colaboradores más importantes destacaban Bagaría, que cobraba 300 pesetas/mes por dos caricaturas a la semana y que fue pronto despedido por no ser abiertamente germanófilo, y los críticos de arte y teatro Tomás Borrás y Santiago Vinardell que, a pesar de que figuraban en la lista negra inglesa, franceses e italianos les consideraban aliadófilos.

4 Es cierto que históricamente los términos espía y espionaje han tenido frecuentemente una connotación peyorativa. Sobre el origen etimológico de estos términos y su evolución conceptual a lo largo de la historia, véase el tratamiento que hace Paolo PRETO en I servizi segreti di Venezia, Milano, Il Saggiatore, 1994, que desarrolló posteriormente en su artículo "Le parole dello spionaggio» publicado primero en la Revista Lingua 
Introducción: Hacia una «cultura de los Servicios...

sia» a este tipo de prácticas, lo más que podría dar el país, siguiendo también una inveterada tradición, serían pícaros, que aparecerían así como la genuina fórmula ibérica del espionaje. En último término venía a descalificarse la propia actividad del espionaje considerándola una especie de "gran montaje» para el aprovechamiento personal de unos cuantos: $\mathrm{Si}$ se niega la premisa mayor, no tiene sentido discutir el resto.

$\mathrm{Al}$ margen de la lucha de propaganda que los beligerantes de la Primera Guerra Mundial mantuvieron en la prensa española, mayoritariamente comprada por uno $u$ otro bando, sabemos que durante aquellos años centenares de agentes extranjeros, con el apoyo de un numeroso grupo de españoles, convirtieron a España en un segundo y enorme frente de combate donde, en último término, estaba en juego la supervivencia del esfuerzo bélico de uno y otro bando.

La Primera Guerra Mundial otorgó carta de naturaleza a los servicios de información o servicios de inteligencia o secretos, etc, pues adoptaban dependiendo del país diversas acepciones. Al igual que sucedió con la propia experiencia bélica, también en el terreno de los servicios de información se fueron planteando progresivamente toda una serie de necesidades que hubo que resolver sobre la marcha, teniendo en cuenta la escasa experiencia previa en este tipo de retos. Nunca como entonces en época contemporánea se habían dedicado tantos hombres y tantos recursos a las actividades secretas. Nunca como entonces nadie se había planteado el «dominio» de un país de las dimensiones de España, entendiendo como tal la infiltración en el tejido básico de la vida de la nación: desde el control de la prensa hasta el establecimiento de contactos al más alto nivel político, económico, y financiero, pasando por la intervención en los centros neurálgicos de la producción industrial, materias primas y alimentos y el control también de las redes de transporte. ¿Conocían los responsables políticos españoles todas las actividades clandestinas que se desarrollaban en el territorio? ¿Crearon los gobiernos de España algún instrumento de control de estas actividades, no ya en pro de la repetidamente violada neutralidad que hubiera sido razón suficiente, sino de la propia seguridad del país? ¿se plantearon "conocer para prevenir, detectar y neutralizar agresiones sobre los intereses propios»? ${ }^{5}$.

Nostra (Diciembre 1995) y recogido más tarde en Per Aspera ad Veritatem. Rivista di intelligence e di cultura professionale, $\mathrm{n}^{\circ} 6$ (1996), en (16/12/2004) http://www.sisde.it/ sito/Rivista6.nsf/stampe/5.

${ }^{5}$ Definición de contrainteligencia que tomo prestada de Francisco Galvache. 
Hablando en términos que no son propios para aquellos años, posiblemente el problema radicase en la percepción de la amenaza. Es decir, que los sucesivos gobiernos de España no consideraran como un peligro para la seguridad -al menos durante un largo período de la guerra- las actividades clandestinas de los beligerantes, a pesar de que algunas de ellas -las encabezadas por alemanes y austríacos- sí tuvieran como principal objetivo la interrupción de la producción española y el sabotaje de las líneas y medios de transporte. En consecuencia, podría soportarse desde el Gobierno y desde la Jefatura del Estado el rumor permanente sobre las actividades secretas extranjeras, pero otra cosa muy distinta era que estas se hicieran públicas en la prensa aportando pruebas documentales entregadas, lógicamente, por el servicio secreto contrario. Es muy significativo en este sentido que la conocida como «Ley Antiespionaje», se redujera en la práctica a facultar al Gobierno para intervenir - censurar o prohibir- todas aquellas publicaciones y noticias que se considerasen a su arbitrio atentatorias contra los países beligerantes o sus altas personalidades, diplomáticas o políticas ${ }^{6}$. Como si ocultar la publicidad de las acciones de las redes de espionaje y contraespionaje condujera a su control o a su desaparición.

La última vez antes de la Primera Guerra Mundial que España organizó -es una manera de hablar- una red de espionaje en defensa de sus intereses fue durante la guerra contra los Estados Unidos en 1898. Entonces, el Agregado Naval en la Embajada de España en Washington, el joven Teniente de Navío Ramón Carranza (alias Frederik W. Dobson), se instaló en Montreal una vez que se declaró la guerra con la intención de crear una red de espionaje pensada para actuar directamente en Estados Unidos. Por distintas circunstancias -alguna ciertamente rocambolescael intento quedó muy lejos de obtener éxito. Sin duda en ello influyó la propia brevedad de la guerra, lo intempestivo del intento y la inexperiencia en tales menesteres del responsable de la red, al que en algunos medios se ha definido injustamente como «one of the most inept spymasters in espionage history, a comical figure really who had no idea of how to accomplish his mission» ${ }^{7}$. Sabemos que de las experiencias bélicas su-

\footnotetext{
6 "Ley dotando al Poder Público de facultades indispensables para garantizar la neutralidad de Españan, 7 de julio de 1918, publicada en la Gaceta de Madrid el 8 de julio, a. CCLVII, t. III, $\mathrm{n}^{\circ} 189$, p. 81.

7 Vid. http://www.angelfire.com/dc/1spy/Carranza.html (1/12/2004) Al contrario, para una versión positiva de Carranza y de su trabajo de espionaje, vid. A.R. RODRÍGUEZ GONZÁLEZ, «El espionaje español en la guerra de Cuba», en Revista Española de Defensa,
} 
cedidas entre finales del siglo XIX y principios del siglo XX, en algunos países - caso de Estados Unidos o de Gran Bretaña- surgieron los proyectos de crear unas nuevas estructuras de servicios secretos que respondieran a las carencias y a las nuevas necesidades que, precisamente, se habían detectado en aquellos momentos de particular peligro para el país. No fue ese el caso de España, ni tampoco puede afirmarse por el momento que se produjera después del Desastre ninguna presión por parte de la opinión pública ni, en concreto, de la opinión especializada (militar, por ejemplo)

En el debate en la prensa por los escándalos del espionaje durante la primavera y comienzos del verano de 1918 no se detectó tampoco ninguna iniciativa que fuese encaminada a presionar al Gobierno para que, a imitación de los beligerantes, creara una estructura, un servicio, que sirviera para controlar las actividades extranjeras en suelo español. En el momento álgido del affaire Bravo Portillo, sólo el Diario de la Marina llamó la atención de sus lectores sobre la desairada posición en la que quedaba España ante el mundo, el ridículo en el que incurría un Gobierno incapaz de tomar las medidas que requería una situación tan peligrosa:

"(...) Impelidos por la acción tenaz y violenta de los alemanes en nuestra patria, España ha llegado a convertirse en un frente de batalla donde los beligerantes luchan con todo denuedo. Frente al espionaje germano se ha opuesto el contraespionaje de los aliados, y ya empezamos a sentir las consecuencias de la lucha. Ahí está el caso Bravo Portillo, y tras él, esperando el momento oportuno para manifestarse, otros casos que nos colocarán en postura difícil y ridícula ante el mundo (...) Vamos a quedar moralmente por los suelos (...) La desidia gubernamental y su desorientación ha hecho posible que esté en peligro nuestra neutralidad habiéndose podido evitar este daño simplemente con una acción diligente y digna de policía (...)».

\footnotetext{
$\mathrm{n}^{\circ} 127$ (septiembre, 1998), pp. 62-63. Este autor afirma que «el proyecto principal era el suministrar información para el raid de la escuadra de (Almirante) Cámara sobre las costas americanas». Para una historia equilibrada y pormenorizada de estos acontecimientos, Rhodri JEFFREYS-JONES, Historia de los servicios secretos norteamericanos, Barcelona, Paidós, 2004, en particular el capítulo «¿Desmanteló Wilkie la red de espionaje de Montre al?», pp. 75-92. Construye la historia a partir fundamentalmente de las publicaciones que realizara el entonces jefe del servicio de inteligencia de los Estados Unidos, John E. Wilkie. En opinión del autor, la consecuencia más destacable de este breve y limitado capítulo de la guerra hispano-norteamericana, fue que provocó en los servicios secretos de los Estados Unidos su transición «de la era del pinkertonismo privado a la del espionaje público».

8 «Nuestra situación ante el mundo. El espionaje y el contraespionaje», en Diario de la Marina, 26 de junio 1918.
} 
Hay datos que demuestran que al principio de la guerra el Gobierno español atendió peticiones que provenían de la Embajada de Francia para que se vigilara a determinados personajes alemanes sospechosos de estar incursos en labores de espionaje. Una vez que los beligerantes crearon sus propios servicios fueron ellos los que actuaban contra las redes enemigas, con la condición sabida de no levantar escándalos que supusieran una alteración del orden o la elevación de una formal protesta diplomática. Sabemos también que si hubo destacados policías que colaboraron con los alemanes, entre los cuales Bravo Portillo sería el más conocido, también los hubo que trabajaron para los aliados y no sería de extrañar que esta actuación permitiera como consecuencia que los Gobiernos de España estuvieran al tanto, con bastante aproximación, de las estructuras y actividades de unos y otros. Posiblemente, ir más allá hubiera supuesto tomar una serie de medidas que implicaban, en primer término, contar con gobiernos estables y políticamente fuertes, que no fue el caso, capaces de correr el riesgo de ver sometido al país a represalias de incalculables consecuencias: fundamentalmente la interrupción del tráfico comercial y el desabastecimiento de productos esenciales. Por lo tanto se creó una especie de acuerdo tácito que, no sin sobresaltos y multitud de problemas, permitió posiblemente a España carecer de un servicio de información al nivel que desarrollaban los beligerantes.

Volvamos a la cuestión que mencionábamos más arriba, ¿Se percibía en España alguna amenaza? ¿dónde radicaba? Aunque todos los apuntes realizados hasta ahora son hipótesis en fase de investigación, es muy probable que la amenaza percibida por los gobiernos de España radicase fundamentalmente en el miedo a la revolución, en la posibilidad de que uno de los problemas que la guerra había contribuido a generar, la revolución rusa, encontrase terreno abonado en una España políticamente inestable y socialmente convulsa. Los sucesos del verano de 1917 eran interpretados como un serio aviso del peligro que corría no sólo el Gobierno de turno sino la propia monarquía española. Si a ello añadimos que sobre todo a partir de 1918 comenzaron a aparecer en España individuos de origen ruso que unían a esta condición, muchos de ellos, el hecho de ser hebreos, la amenaza, ahora sí, se percibía con absoluta certeza porque ya se había instalado en la Europa occidental el lugar común que establecía una relación directa entre los judíos rusos y el hecho revolucionario.

La respuesta no se hizo esperar, finalizada la guerra, en diciembre de 1918 el Gobierno encabezado por Romanones decidió crear un cuerpo de «policía especial» denominado Escuadra Volante Secreta formado por cincuenta hombres bajo el mando del comisario Francisco Martorell, exper- 
to en la lucha antianarquista, que se había destacado durante la guerra por sus enfrentamientos con Bravo Portillo, por pasar información a los aliados -supuestamente de forma secreta- y por su vocación personal aliadófila. Romanones buscó el apoyo de los servicios secretos aliados que habían estado operando en España, para hacer frente a un peligro que se consideraba «global», no específicamente español y que, en consecuencia, involucraba a todos en un momento histórico tan delicado por el efecto de «contagio» transfonterizo que se le atribuía. Muy pronto los propios aliados, no ya solo la propia opinión pública española, percibieron que la actuación de esa unidad "secreta» española tendría muy poco que ver con la forma de actuar de un servicio secreto propiamente dicho, pues a la información y control de los movimientos de extranjeros en suelo español uniría también la detención y el uso de métodos que, como afirmó el jefe del servicio italiano, «sospecho que sean un poco demasiado españoles de la inquisición» ${ }^{9}$.

Con las páginas que preceden he intentado ilustrar un momento fundamental de la historia de los servicios secretos. Según opinión admitida generalmente, se trataría del período y la coyuntura concreta -la Primera Guerra Mundial- que está en el origen de los servicios del siglo XX. Es paña, sujeto paciente de ese surgimiento a gran escala de los servicios se cretos no se incorporó a esta nueva modalidad estructural de obtención de información para elaborar, como se diría hoy, inteligencia. He tratado en someras notas apuntar la razón fundamental pero, desde luego, no fue la única. La modesta presencia internacional de España, su lejanía de los puntos de conflicto candentes en la Europa de aquellos años o, dicho de otra forma, la inexistencia de compromisos internacionales «ejecutivos»

\footnotetext{
9 Archivo del Ufficio Storico della Marina Militare, Legajo 1277, Stato Maggiore. Despacho $\mathrm{n}^{\circ} 17152$, del Agregado Naval de Italia en Madrid al Ufficio $I V$ del Jefe de Estado Mayor del Ministerio de la Marina, Madrid, 13 de diciembre de 1918. Dos días antes de escribir estas líneas, el Agregado Naval había recibido una nota en la que Francisco Martorell le decía que el súbdito ruso de nombre Wladimir Tinikof (alias Michel Weissben), que el día anterior era conducido a Barcelona para ser expulsado de España por orden gubernativa, había muerto al intentar escapar en Zaragoza como resultado de los disparos de la policía que le custodiaba. La nota oficial que fue dada a la prensa decía, sin embargo, que había muerto al tirarse del tren en el que viajaba. Por esta razón el Agregado habla en su informe de los métodos usados en España y finaliza su escrito asegurando que, en adelante, «irán con pies de plomo» en su colaboración con los españoles para no «tener siquiera la más lejana sospecha de ser copartícipes en injusticias de ningún tipo».
} 


\section{Fernando García Sanz}

que pudieran arrastrarla en una hipótesis bélica, posibilitó quizás que Es paña pudiera seguir viviendo con dosis mínimas o nulas de información al margen de los canales tradicionales: los despachos de los agregados militares y de los diplomáticos de carrera. Sin querer aquí entrar en el debate sobre la primacía política exterior/política interior, sí es cierto que durante muchos años ha prevalecido en España el peso de una percepción interna de los conflictos europeos, parafraseando el famoso ensayo de José $\mathrm{M}^{\mathrm{a}}$ Jover $^{10}$. Los servicios secretos se han visto también afectados por este hecho a lo largo del tiempo. Salvando el lapso de la Guerra Civil, cuando se activa una modalidad tradicional -táctica- de las actividades de información, el resto del período, hasta bien entrada la transición, la función de las actividades secretas se dirigieron fundamentalmente -por lo que sabemos hasta ahora- a desactivar todo acto de subversión interna: la percepción de la amenaza se centraba por tanto durante la dictadura en la seguridad del régimen político y su continuidad.

El hecho de titular este monográfico Al servicio del Estado: Inteligencia y Contrainteligencia en España, no proviene de la voluntad de resaltar lo evidente. Es sobre todo, a mi juicio, el resultado tanto de una declaración de intenciones como del reconocimiento de un determinado, inmediato y difícil desenvolvimiento histórico. Para la inmensa mayoría de los ciudadanos la percepción de los servicios de inteligencia, deformada también por el éxito que estos temas han alcanzado siempre en cine y literatura, es el resultado de una mezcla de desconfianza y fascinación. Es raro el país en el que los servicios secretos no han despertado -y despiertan todavía- la suspicacia cuando no la abierta desconfianza de los ciudadanos ${ }^{11}$. Por ello, hay que preguntarse por las razones que han hecho que estas actividades estén siempre bajo sospecha, que incluso hayan podido llegar a ser concebidas más como una fuente de problemas que como un buen recurso para ser utilizado en pro de los intereses nacionales definidos legítimamente por un Estado democrático. Habría dos tipos de argumentaciones, unas de tipo genérico que incumben al propio tipo

10 «La percepción española de los conflictos europeos: notas históricas para su entendimiento", en Revista de Occcidente, $\mathrm{n}^{\circ} 57$ (1986), pp. 5-42.

11 Algunos ejemplos a este respecto, Brigitte HENRI, Le Reseignement. Un enjeu de pouvoir, Paris, Éditions Economica, 1998; Giuseppe DE LUTIIS, Storia dei servizi segreti in Italia, Roma, Editori Riuniti, 1991; Rhodri JEFFREYS-JONES, Historia de los servicios secretos norteamericanos, op. cit.,; Mario CALIGIURI, «Intelligence: un problema di comunicazione istituzionale?», en Per Aspera ad Veritatem, $\mathrm{n}^{\circ} 16$ (2000), en http://www.sisde.it/sito/Rivista16.nsf/servnavig/4 (07/12/2005). 
Introducción: Hacia una «cultura de los Servicios...

de actividad -secreta- que realizan estos servicios, las otras más concretas que tendrían directa relación con la particular historia de cada uno de los servicios o, mejor aún, con la historia de cada país. En algunos casos porque los servicios se han visto envueltos en oscuros escándalos que no han sido suficientemente aclarados y que han puesto en tela de juicio el uso que los gobiernos hacían de instrumento tan delicado; en otras ocasiones porque, desgraciadamente, la propia índole de su trabajo conlleva que sean «visibles» en los fracasos, no en los éxitos. En el caso español se incrementarían en justicia las prevenciones hacia los servicios secretos teniendo en cuenta nuestra historia durante buena parte del siglo XX.

El caso español es particular dentro del panorama europeo por haber padecido un régimen político dictatorial durante tantos años una vez que fueron derrotados los regímenes fascistas. En este sentido, en su colaboración para este monográfico, el General Francisco Javier Zorzo nos ilustra sobre este rasgo distintivo de la situación española de aquellos años haciendo hincapié fundamentalmente en los aspectos organizativos y los objetivos de los servicios de inteligencia españoles. Sin embargo, no deja de poner en evidencia que el mismo clima de guerra fría que introdujo a España en el marco estratégico de la defensa occidental, de la mano de los acuerdos con los Estados Unidos, sirvió también para que oficiales españoles fuesen enviados a este país y al Reino Unido «para recibir formación específica en el campo de la información y se comienza a enviar a agentes españoles al extranjero, marchando, durante bastante tiempo, de la mano de la CIA». Aún así, subraya Zorzo, los agentes que se comenzaron a desplegar en el extranjero, ya desde la inmediata postguerra bajo la dirección de Gutiérrez Mellado, tenían como tarea principal el control de las actividades de los exiliados. Pero también el caso español es particular, porque los propios servicios secretos de la dictadura se vieron en la circunstancia de tener que colaborar en la transición política hacia un régimen democrático. Un cambio de funciones copernicano, por tanto muy delicado y complejo, ya que a partir de entonces se pasaba de impedir la oposición al régimen a garantizar que todas las fuerzas políticas y sociales pudieran comenzar a ejercer una serie de derechos que quedarían finalmente sancionados por la Constitución de 1978.

El General Juan María de Peñaranda con su trabajo sobre «los servicios de inteligencia durante la transición» destaca fundamentalmente tres aspectos. En primer lugar que los tiempos de la transición política posiblemente no coincidan con los de la transición de los servicios de inteligencia, pues el arranque de los cambios en este aspecto habría que retrotraerlo a las transformaciones que se comienzan a operar a partir de 
1968 y que, desde su punto de vista, sirvieron para que España pudiera buscar el rumbo de aproximación al resto de los países occidentales. En segundo lugar, Peñaranda, como desarrollo lógico del anterior aspecto, defiende el papel relevante de los servicios españoles (el Servicio Central de Documentación -SECED-fundamentalmente) en la evolución de una pacífica transición política, mediante la activación de una serie de operaciones específicas (Lucero, Alborada y Relámpago) elaboradas de acuerdo a cada uno de los principales cambios que se preveían: «Ni el tránsito a la democracia hubiera podido resultar exitoso sin los Servicios, ni estos se hubieran desarrollado como convenía si no se hubiera producido la Transición». En tercer lugar, creo que es importante el destacado papel que otorga a las personas, sobre todo a los entonces Tenientes Coroneles Valverde y Cassinello, los dos últimos responsables del SECED, no solo por considerarles protagonistas del cambio pacífico sino, sobre todo en el caso del último de ellos, porque fue capaz en la nueva etapa que se abría de comenzar a inculcar en los servicios el espíritu apartidista, el sentido de Estado «que diferenciaba la acción del Gobierno Suárez de la correspondiente al partido político que le sustentaba» ${ }^{12}$.

Es difícil abordar en espacio tan reducido la historia del Centro Superior de Información de la Defensa (CESID) como hace en estas páginas Carlos Ruíz Miguel. El nuevo servicio de inteligencia y contrainteligencia, último de la etapa preconstitucional y primero de la etapa democrática, tuvo que enfrentarse a problemas específicamente españoles, como el control de posibles amenazas militares internas y el terrorismo de ETA, y a los retos que en cualquier Estado democrático genera el trabajo de estos servicios y el encaje y control legal, político y parlamentario de las actividades que les son propias ${ }^{13}$. Ruíz Miguel pone en evidencia que si, por un lado, el CESID debe ser considerado como el primer servicio de inteligencia español homologable a los de los países de nuestro entorno (sobre todo cuando a partir de 1982 asume por primera vez misiones de inteligencia exterior y tareas de contrainteligencia), su articulación dejó sin resolver sin embargo algunas cuestiones fundamentales que abordaría la Ley que

12 Esperamos con sumo interés la publicación de la Tesis Doctoral del General Peñaranda sobre los servicios de inteligencia durante la Transición porque, sin duda, profundizará en todos los importantes aspectos que aborda en su colaboración para este monográfico.

13 Precisamente sobre este tema, Ruíz Miguel es uno de los mayores expertos es pañoles. Como ejemplo, véase su obra Servicios de inteligencia y seguridad del estado constitucional, Madrid, Tecnos, 2002. 
Introducción: Hacia una «cultura de los Servicios...

amparó la posterior creación del Centro Nacional de Inteligencia. En este sentido me resulta particularmente significativo el epígrafe «el guardaespaldas maniatado» en tanto en cuanto refleja una cuestión que no ha dejado de estar en permanente debate en la literatura internacional al respecto y, me atrevería a decir, que se sitúa en el centro del debate en la opinión pública sobre las actividades de los servicios de inteligencia. Es decir, siendo la misión básica de los servicios obtener información para elaborar inteligencia, ¿cómo se obtiene esa información?; en segundo lugar, siendo obvio que no toda la información es abierta y por tanto accesible por métodos «convencionales», ¿cómo se «legalizan» los métodos «ilegales» en un Estado democrático?, ¿la ilegalidad es inherente a las actividades secretas? En 1997 una revista italiana reunió a una serie de expertos internacionales con la intención de que respondieran a la pregunta a che servono $i$ servizi? De las distintas respuestas me parece particularmente significativa, simple y clara, la que aportó el Almirante Gianfranco Batteli, Jefe durante cinco años (1996-2001) del Servizio per le Informazioni e la Sicurezza Militare (SISMI) ${ }^{14}$ :

"Estoy convencido de que si un Estado decide dotarse de Servicios de Inteligencia es porque ha llegado a la conclusión de que con los medios ordinarios no le es posible garantizar adecuadamente la seguridad. Pedir que operen en el ámbito de la legalidad que hace de marco a la actividad de la magistratura y de las fuerzas de policía es, en consecuencia, una auténtica contradictio in terminis, que termina por negar la propia razón de la creación de servicios de información y seguridad. Me parece, por tanto, demasiado obvio que los servicios deban poder hacer cosas ilegales».

La conclusión del trabajo de Ruíz Miguel se incardina precisamente en este aspecto, en la resolución ayer (CESID) como hoy (CNI) de esta paradoja que incumbe al resultado del trabajo de los servicios de inteligencia: «su funcionamiento debe ser secreto, pero debe estar regulado y garantizado por las instituciones públicas».

¿Cómo han sido vistas las vicisitudes de nuestros servicios de inteligencia a lo largo del tiempo por nuestros historiadores? Esta pregunta, metodológicamente básica para cualquier historiador que se propone iniciar una investigación, se la hemos realizado al investigador del CSIC Juan Goberna Falque. Su respuesta, que abre este monográfico y que obligatoriamente se ha visto constreñida por el limitado espacio a dispo-

14 «A che servono i servizi», en LIMES, nº 3 (1997), pág. 293. 


\section{Fernando García Sanz}

sición, creo que viene a completar satisfactoriamente la primera parte, de las tres en las que hemos dividido este monográfico, dedicada a la historia de nuestros servicios de inteligencia. Aunque la abundancia de títulos y de temas es sorprendente, Goberna nos aclara que su trabajo es solo una primera aproximación al tema, que tiene el beneficio -y los inconvenientes- de la novedad, pero que tendrá continuidad en un próximo futuro y muy probablemente en forma de monografía. Desde el punto de vista del autor, la gran cantidad de títulos de los que se hace eco no responden, sin embargo, a un trabajo sistemático, no constituyen, por tanto, una línea historiográfica propiamente dicha. En segundo lugar, sólo a partir de los años de la transición comienza a ser constante la aparición de títulos dedicados al tema aunque el auténtico boom se haría esperar hasta la década de los noventa cuando «la oleada de escándalos en los que se ven envueltos los servicios secretos (...) posibilita que estos pasen a ocupar por primera vez en la historia de la España contemporánea, y por un tiempo largo además, el centro de la escena mediática». En tercer lugar, este mismo hecho ha traído consigo que una buena parte de las publicaciones no sean debidas a historiadores, sino a periodistas, argumento que aprovecha Goberna para apuntar un debate cuyas consecuencias deja al juicio del lector: periodismo e historia mantienen una relación distinta con el tiempo, con los hechos y con los métodos de análisis de tal forma que «la mezcla de géneros mantiene la confusión e impide desempeñar convenientemente cada una de las funciones de las que la sociedad tiene necesidad». Desde luego será esta una situación que se mantendrá en el tiempo mientras las fuentes de información -documentales, para realizar el trabajo que le es propio- permanezcan lejos del alcance del historiador. La ecuación es sencilla, el trabajo de investigación de los historiadores es similar al de los servicios secretos: no elaborarán inteligencia sin información o elaborarán mala inteligencia con escasa o mala información.

Un segundo bloque de este monográfico viene constituido por tres colaboraciones destinadas a poner de relieve la actualidad de los servicios de inteligencia y contrainteligencia centralizados desde 2002 en el CNI, sustituto cronológico del CESID. En el primero de ellos, Rafael Jiménez lleva a cabo una forzosamente apretada síntesis sobre las características principales del CNI. El profundo cambio en las relaciones internacionales que se produjo a principios de los años noventa junto a la necesidad de subsanar las deficiencias detectadas en el funcionamiento del CESID ("escándalo Perote») llevaron a la conclusión de que el instrumento de inteligencia creado en 1977 ya no era válido. Se impuso entonces al poder 
Introducción: Hacia una «cultura de los Servicios...

ejecutivo la tarea urgente de llevar a cabo una reforma que fuera capaz, como una de sus tareas prioritarias, de elaborar un marco legislativo que garantizase tanto los derechos constitucionales del ciudadano como que amparase al Centro «y, en particular, a sus miembros, en el cumplimiento de algunas misiones, durante cuya ejecución habían quedado desprotegidos». Jiménez nos ofrece un esquema explicativo en el que, poniendo de relieve las novedades del nuevo aparato de inteligencia tanto en relación con sus antecedentes españoles como con sus homólogos occidentales, hace un repaso al marco legislativo, a los objetivos, las misiones, el sistema de trabajo, selección y características del personal que trabaja en el CNI, subrayando en último término que -permítaseme la broma- cualquier español, a diferencia de lo que señala el texto con el que se abren estas páginas, puede ser espía si reúne una serie de condiciones que le llevarán a desempeñar uno u otro puesto pero igualmente útil en la elaboración de inteligencia. El CNI es pariente del final de la guerra fría, pero hijo directo del ataque a las torres gemelas de Nueva York el 11 de septiembre de 2001. Hasta tal punto, nos señala el autor, que influyó «en el tratamiento de sus principios básicos (del CNI) y en el ritmo aplicado a los trabajos técnicos y parlamentarios». Desde este punto de vista -la percepción clara de una de las mayores amenazas, el «terrorismo internacional»-, una estructura dotada con los medios necesarios y bajo el triple control político, parlamentario y judicial, podría proyectarse hacia el futuro con mayores garantías que en el pasado de conseguir los fines para los que fue creado. El reto actual, sin embargo, como no deja de esbozar Rafael Jiménez, está en conseguir una mayor optimización de los recursos de todos los servicios de información al servicio del Estado mediante la necesaria coordinación de los mismos.

De acuerdo en que la coordinación de servicios -no sólo los nacionales- es una de las necesidades más urgentes, acuciante desde los atentados del 11 de marzo de 2004 en Madrid, solicitamos a Francisco Galvache Valero que nos hiciera una puesta a punto de esta importante cuestión. Ya en el título del artículo encontramos algunas claves: que no existe una "Comunidad de Inteligencia» y que aunque está en "marcha» hay que pasar por un "proceso». De forma muy didáctica Galvache nos conduce al objetivo de su artículo partiendo de la explicación de los propios conceptos (inteligencia, contrainteligencia, seguridad, etc), razonando sobre el contexto general (el escenario, los riesgos y la amenazas, etc) y buscando en el proceso del "ciclo de inteligencia» los puntos en los que una "comunidad de inteligencia»-que interpreta como única salida para hacer frente a las amenazas- encontraría más fácilmente la forma de ha- 
cer encajar los engranajes para un correcto funcionamiento. Se puede aducir que este es un viejo debate, tanto al menos como la propia historia de los servicios de información, pero creo que el objetivo de nuestro autor, mediante la estructura que ha dado a su trabajo, es remarcar precisamente que el momento actual es distinto a todo lo vivido anteriormente y que, en consecuencia, las disfunciones que en otros momentos podían sobrellevarse ahora resultan ya intolerables. $\mathrm{Y}$ dentro de este marco hay que detenerse en un punto que Galvache considera imprescindible, tanto o más importante que otras de las fórmulas que aporta para la creación de una "comunidad de inteligencia», pues es dentro de ese ámbito en el que hay que situar éste número monográfico de la $\mathrm{Re}$ vista Arbor. Me refiero a la necesidad que señala de crear una «cultura de la inteligencia», que los servicios ("sin menoscabo de la seguridad») se abran a la sociedad porque deben apoyarse en ella para desarrollar con éxito su labor, porque solo de esta forma se podrá evitar, concluye Galvache, «uno de los males más peligrosos para un servicio de inteligencia: el ensimismamiento, la desconexión con el referente contextual que aísla y conduce al pensamiento circular y acrítico». Este debate sobre la creación de una "cultura de la inteligencia» se presenta como una novedad en España, pero no lo es en los países de nuestro entorno que llevan ya varios lustros sobre el tema, conscientes de las posibilidades que se abren volcándose en establecer un dialogo permanente con la sociedad que tendría como primera consecuencia comenzar a cambiar la histórica percepción del ciudadano sobre sus servicios de inteligencia y, cambiando las mentalidades, permitir que los servicios de inteligencia ocupen el lugar que le corresponde en la sociedad ${ }^{15}$.

A diferencia de lo que ocurría hace apenas tres décadas, España también ha conocido importantes transformaciones en sus relaciones internacionales, ha asumido un papel importante en aquellos foros en los que está presente y ello ha obligado también a que los servicios de inteligen-

\footnotetext{
15 Breve y esclarecedora exposición de los beneficios de la creación de una «cultura de la inteligencia» y los problemas a los que se enfrenta en Pierre LACOSTE, "Cultura e intelligence: un progetto per l'Università", en Per Aspera ad Veritatem. Rivista di intelligence e di cultura professionale, $\mathrm{n}^{\circ} 6$ (1996), en http://www.sisde.it/sito/Rivista6.nsf/stampe/5 (16/1/2005) El autor, Almirante y responsable de la Direction Generale de la Securite Exterieure (DGSE) entre 1982-1985, consideraba ya entonces que en este terreno los países anglosajones se encontraban mucho más avanzados que, por ejemplo, franceses e italianos. Me resulta particularmente significativo que Lacoste subraye la necesidad de hacer frente a los problemas que plantean los historiadores, que tienen a su disposición pocas fuentes, y de acabar con una exagerada "cultura del secreto».
} 
Introducción: Hacia una «cultura de los Servicios...

cia tuvieran que ampliar su radio de acción convirtiendo su rama exterior, por primera vez en la historia contemporánea de España, en un instrumento al nivel del protagonismo internacional del Estado y del incremento de los intereses nacionales en las más diversas regiones del planeta. Federico Torres Muro, diplomático y experto en inteligencia, analiza esta cuestión comenzando por apuntar las transformaciones ocurridas en la comunidad internacional desde la caída del muro y como el panorama internacional, lejos de simplificarse, se ha complicado sobremanera. Los servicios de inteligencia, señala Torres, tienen que hacer frente a nuevos «fenómenos transnacionales de alto potencial desestabilizador» y moverse en una sociedad internacional que ha visto crecer el número de sus actores no estatales llegando a transformarla y hacerla más compleja. Dentro de este contexto general, Torres analiza las prioridades de la acción exterior de España (Unión Europea, Mediterráneo, Iberoamérica, relación transatlántica) poniendo de relieve en cada uno de los apartados el papel que le corresponde desarrollar a los servicios de inteligencia en cada una de las áreas de interés de la política exterior de España. Responsabilidades muy diversas que irían desde la lucha contra la inmigración ilegal, el terrorismo, el narcotráfico, el apoyo a la estabilidad democrática en el ámbito iberoamericano y a la defensa de los intereses económicos españoles, la ayuda a la consolidación de la Autoridad Nacional Palestina ("contribución no muy conocida») o, en fin, la formación de un ámbito de seguridad europeo que no solo no sea incompatible, sino que venga a fortalecer los lazos de los aliados europeos en el seno de la OTAN.

El tercer y último bloque de este monográfico está dedicado a abordar lo que hemos denominado como «los nuevos retos», es decir aquellos nuevos problemas de presente y de futuro a los que se enfrentan los servicios de inteligencia y que ya no aparecen como específicamente españoles, sino más bien inherentes a las actividades de inteligencia en los países democráticos.

Jordi Marsal Muntalá, desde la atalaya del Congreso de los Diputados, reflexiona sobre los conceptos seguridad/libertad como objetivos inescindibles de una misma labor: «los servicios de inteligencia son servicios del Estado, son un instrumento más que el Gobierno tiene para garantizar la seguridad y, por ello, para garantizar las libertades y el ejercicio de los derechos por parte de los ciudadanos y con ello garantizar la democracia del país». En torno a esta cuestión, Marsal sitúa el debate en la actualidad en la tensión que puede generarse entre una mayor garantía de la seguridad con la aplicación de unas determinadas medidas 


\section{Fernando García Sanz}

-principalmente las antiterroristas- que puedan llegar a coartar el ejercicio pleno de las libertades inherentes a un país democrático. Considera el autor que en el caso español el sistema legal adoptado para el control de las actividades de los servicios de inteligencia, aun siendo más "garantista" que en otros países europeos, no parece que vaya en detrimento de su eficacia, siendo conscientes -concluye Marsal- que en una sociedad abierta «la seguridad nunca es total».

El mayor reto al que se enfrentan en la actualidad los servicios de inteligencia occidentales es hacer frente al terrorismo que en este mismo volumen encontramos descrito con diversos nombres: internacional, islamista o yihadista, como hace, en este ultimo caso, Javier Jordán Enamorado en su colaboración sobre «los servicios de inteligencia y la lucha antiterrorista». El resultado es una exposición esclarecedora que, a pesar de la obligatoria brevedad de espacio, no renuncia a tratar con gran maestría -sobre todo desde el punto de vista de un profano como el que firma estas líneas- los aspectos fundamentales de la cuestión. En primer lugar, comienza por razonar y explicar el concepto que utiliza, terrorismo yihadista, sus presupuestos ideológicos, medios operativos y objetivos ("se trata de un empleo de la fuerza dirigido a destruir, derrocar o expulsar a los etiquetados como enemigos (...) sin mayores límites a la violencia que aquellos que imponen los medios materiales y los condicionantes operacionales a la hora de llevarla a cabo») y, en fin, su organización, también con respecto a los modelos de otros grupos terroristas, y su variación en el tiempo teniendo en cuenta como hecho fundamental el atentado de Nueva York. Los servicios de inteligencia necesitan adaptarse a esta gran amenaza distinta a otras conocidas y, por tanto, necesitada de respuestas nuevas. No solo los servicios de inteligencia, sino en primer lugar los estados, han necesitado un período de tiempo para percibir este tipo de terrorismo como una amenaza global y ello no se ha producido -subraya Jordán- hasta que algunos estados no se han visto afectados individualmente por las consecuencias de la amenaza. Este gran problema es tratado en la segunda parte del artículo mediante el análisis de la necesaria adaptación del ciclo de inteligencia a esta amenaza concreta concluyendo, de la misma forma que hacen otros autores de este volumen, que el último escalón del ciclo -la difusión-implica la existencia y un buen funcionamiento de la comunidad de inteligencia «entendida como la estructura de trabajo permanente entre los distintos organismos del Estado relevantes desde el punto de vista de la inteligencia y no sólo el conjunto agregado de estos». La forma en la que afrontó, afronta y debería afrontar el CNI la amenaza de este tipo terro- 
Introducción: Hacia una «cultura de los Servicios...

rismo, antes y después del 11 de marzo de 2004, cubre la tercera parte del análisis que nos ofrece Jordán. De todos los aspectos que son tratados hay que subrayar -también aquí coincide con otros autores en este mismo volumen- el reto concreto para los servicios españoles de crear una auténtica comunidad de inteligencia. En este sentido, valora como un gran paso la creación del Centro Nacional de Coordinación Antiterrorista (CNCA) pero insuficiente si no se da un "cambio cultural entre los protagonistas de la cooperación a todos los niveles». La conclusión en este terreno va en el sentido que apuntara Galvache en su artículo, es decir que las reformas o la creación de nuevos instrumentos de trabajo, fundamentalmente los dirigidos a crear o mejorar la coordinación, no tendrán éxito si el proceso no se ve acompañado de un importante cambio de mentalidades.

El terrorismo yihadista no es la única amenaza global a la que se enfrentan los países en la actualidad. Juan Avilés Farré aborda esta cuestión centrándose en el análisis de tres grandes problemas que no se presentan aíslados, que aparecen muchas veces relacionados entre ellos y también con el fenómeno terrorista: las consecuencias que tiene para la seguridad el incremento de los flujos migratorios, la proliferación de armas de destrucción masiva y el aumento de la delincuencia organizada y del narcotráfico. En cuanto al primero de los problemas, Avilés lo analiza primero en términos generales haciendo hincapié en aquellos aspectos de las actuales características de los flujos migratorios que pueden afectar más directamente a la seguridad, para pasar a exponer la influencia de esta cuestión en el concreto caso de España. Fundamentalmente la falta de integración en los países de destino, ya sea por el rechazo a los valores de la nueva sociedad, ya sea por la situación de precariedad, ya por el incumplimiento de las aspiraciones, estaría -según nuestro autor-, en el origen del incremento de la delincuencia y, en lo que respecta a una minoría de inmigración musulmana, en su adscripción a posiciones radicales que pueden finalizar en la comisión de acciones terroristas como se puso dramáticamente en evidencia en los atentados del 11 de marzo en Madrid. En cuanto al segundo de los problemas, Avilés discrimina entre la proliferación del arma nuclear, entre algunos estados que no firmaron el Tratado de No Proliferación (1968) como Israel, India y Pakistán o que lo han roto como es el caso de Corea del Norte, y el tráfico clandestino de tecnología nuclear o radiológica que en un momento determinado podría caer en manos de grupos terroristas. Más accesible y difícil de controlar, por tanto más peligroso, es desde su punto de vista, la posibilidad que tiene el terrorismo de adquirir y usar las armas quí- 
micas y biológicas. En cuanto a la tercera de las amenazas globales, la delincuencia organizada, Juan Avilés destaca sus vinculaciones con el narcotráfico -que aparecería como la principal fuente de ingresos-, la trata de personas, y el tráfico de armas. Nuestro autor concluye con el reconocimiento que existen dos procesos distintos y compatibles para afrontar toda esta problemática, uno dirigido a buscar la fórmula para hacer frente a sus posibles causas (hambre, pobreza, etc), otro dirigido a neutralizar la amenaza, terreno en el que los servicios de inteligencia tienen un papel fundamental que desarrollar. Servicios en plural, pues las amenazas globales reclaman la imperiosa necesidad de ahondar en la cooperación internacional.

La globalización de las amenazas hace que la seguridad de los países esté cada vez mas imbricada. Siendo evidente la necesidad de una mayor cooperación internacional entre los distintos servicios de inteligencia, ¿cómo se lleva a cabo esta cooperación y cuales son los problemas principales para que podamos hablar de una comunidad internacional, al menos europea, de inteligencia? Esta es la cuestión fundamental que aborda Julia Pulido Gragera en su colaboración para este monográfico. Parte la autora por resaltar algunos conceptos básicos de la teoría de las relaciones internacionales que, aunque en buena medida hayan podido verse sobrepasados por el impacto de los acontecimientos, estarían aún hoy presentes en el comportamiento de los estados como una rémora a la hora de definir el concepto de seguridad y, sobre todo, de seguridad compartida. En segundo lugar, Pulido analiza los problemas a los que se enfrenta la cooperación intraestatal entre servicios de inteligencia e información (otra vez la cuestión de la comunidad de inteligencia nacional) y cual es la experiencia en este terreno. Cooperación intraestatal que destaca como un paso previo y necesario para una más útil cooperación interestatal. Ésta es analizada desde el punto de vista bilateral y multilateral. La primera de ellas presentaría menores dificultades, quizás porque es la fórmula más veces ensayada a lo largo de la historia de los servicios de inteligencia y, desde muchos puntos de vista, más fácilmente realizable. La cooperación multilateral resultaría mucho más compleja porque «cada Estado presenta unas necesidades conforme a su seguridad e interés nacional», aún así Pulido demuestra que esta cooperación ha sido posible con anterioridad y desgrana algunos ejemplos de redes de inteligencia (UKUSA, ECHELON, CAZAB), de colaboración en el ámbito de organizaciones multilaterales como la OTAN y de grupos de coordinación internacionales, como el Club de Berna, el Grupo de Trevi, el Grupo Kilowatt o el Grupo de Egmont subrayando las características específicas de 
cada uno de ellos. Por muchas razones el caso de la cooperación en el seno de la Unión Europea presenta características particulares. Sobre todo después de los atentados de Nueva York y Madrid, la Unión, afirma nuestra autora, se ha planteado la necesidad «de un redimensionamiento de los servicios de inteligencia y de información", pero se ha descartado la creación de una "Agencia Europea de Inteligencia» a cambio de la creación de otros órganos de coordinación en los ámbitos militar, estratégico y policial (Europol, Grupo sobre Contraterrorismo, División de Inteligencia adscrita al Estado Mayor de la Unión, etc.). Es factible, concluye Julia Pulido, una mayor cooperación multilateral siendo fundamental para alcanzarla objetivos claros e incrementar la confianza entre los Estados.

Por último, no podíamos dejar de analizar el lugar que ocupan las nuevas tecnologías en el trabajo de inteligencia, partiendo de la base que si bien se constituyen hoy día -y lo serán más en el futuro- una herramienta imprescindible de trabajo pueden convertirse también en una amenaza. Diego Navarro Bonilla aceptó el encargo de reflexionar al respecto ofreciéndonos un denso trabajo que no empaña sin embargo la claridad expositiva. En primer lugar comienza por razonar en torno a toda una serie de consecuencias que tiene la revolución tecnológica aplicada al mundo de la inteligencia: una parte de tecnología que puede usarse, por ejemplo, en la lucha contra las redes terroristas puede, a su vez, ser usada por estas para llevar a cabo sus objetivos; las nuevas tecnologías facilitan y también exigen una mayor coordinación de esfuerzos entre los distintos servicios de inteligencia e información a escala nacional e internacional; las nuevas tecnologías aplicadas a los campos de la Seguridad y la Defensa han facilitado la Revolución en los Asuntos Militares, que cambia radicalmente el concepto de las guerras futuras; en fin, un determinado uso de las nuevas tecnologías puede poner en peligro las libertades civiles «en aras de una absoluta preeminencia de la seguridad». En segundo lugar, nos describe algunas de las modalidades y usos de la tecnología actual (captación de señales, interceptación de comunicaciones, información geográfica digital, obtención de imágenes mediante aviones sin tripulante y satélites, etc) ilustradas con algunos significativos ejemplos y el grado de participación de España en estas nuevas tecnologías. En tercer lugar, analiza la llamada Information Warfare entendida como «el marco global en el que se incluyen no sólo "todos los medios capaces de neutralizar el empleo de redes de ordenadores de un enemigo" sino también todas las operaciones relacionadas con la obtención, procesamiento y uso de la información desarrolladas en un entorno 
real o virtual para alcanzar un objetivo de ventaja predominante sobre un posible competidor económico, político o militar». En cuarto y último lugar, Diego Navarro aborda lo que cada vez representa un mayor problema para los servicios de inteligencia como es la abundancia de información obtenida a través de fuentes abiertas. Un problema que, a su juicio, sólo puede tener solución mediante la formación de expertos en el tratamiento de este tipo de información y que pone en evidencia-viene a concluir Navarro- que las nuevas tecnologías siguen siendo "sólo» un sofisticado instrumento para generar conocimiento pero -como han demostrado también recientes experiencias- no pueden sustituir al «factor humano» en la producción de inteligencia.

Todos los colaboradores de este número monográfico, a los que agradezco sinceramente su magnífico trabajo, son especialistas en las áreas que tratan referidas a los servicios de inteligencia. El único que no puede mostrar tal grado de especialización es el firmante de estas páginas introductorias que han intentado, por otro lado, presentar al lector los aspectos más importantes de los temas tratados a continuación. Interesado por la Historia de la Inteligencia y responsable en la actualidad de una investigación al respecto, asumí con mucho interés el encargo de coordinar este número en la medida que me ofrecía la doble posibilidad de colaborar en el proyecto de hacer socialmente más visible un sector de la administración del Estado de tanta importancia como es el servicio de inteligencia y porque, siendo responsable de trazar la estructura del monográfico, podía hacerme intérprete no sólo de las preguntas que al respecto podían hacerse desde un sector del mundo académico, sino desde la ciudadanía en general.

La contribución de los historiadores a la formación de una «cultura de la inteligencia» se presenta como una tarea compleja, porque -quizás por un exceso en la consideración de lo secreto, quizás por la dificultad de asumir nuestro propio pasado- el acceso a nuestra principal herramienta de trabajo, la documentación, resulta verdaderamente problemático. En la medida en que los historiadores podemos ofrecer una colaboración útil al esfuerzo de abrir a la sociedad el trabajo que realizan los servicios de inteligencia y contrainteligencia españoles, entendemos que esa colaboración debe entenderse como un camino de ida y vuelta, que invita a que -por fin-también los servicios se abran con total normalidad al trabajo de los historiadores. Un mayor conocimiento de la historia de este tipo de actividades solo puede redundar positivamente en una mejor comprensión de la historia de España en su conjunto. 
Introducción: Hacia una «cultura de los Servicios...

Se puede estar de acuerdo con el CNI en que hay que generar y extender una "cultura de los servicios de inteligencia» que acerque al ciudadano una labor tan importante que comience por desterrar los mitos y las deformaciones. Sin embargo, corremos el riesgo cierto de quedarnos en un mero trabajo de propaganda si no damos profundidad al acertado intento investigando nuestra propia historia y presentando al ciudadano el recorrido que en nuestro país han tenido unos servicios tan importantes para entender la propia evolución del Estado a lo largo, al menos, del último siglo. Pero en la perspectiva que a nuestro grupo de investigación más interesa, no se trata sólo de contribuir con el estudio de un aspecto poco tratado al conocimiento de la historia interna de España, sino sobre todo al de sus relaciones internacionales, como un buen termómetro de análisis de la participación de nuestro país en las grandes corrientes de evolución histórica. Por ello, no creemos que dentro de la investigación de la historia de la política exterior y la política de defensa pueda descuidarse por más tiempo la incorporación de parámetros tan importantes como es el de los servicios de inteligencia. El simple hecho de su existencia o no en sincronía con otros ejemplos de nuestro entorno europeo; las características de su estructura y funcionamiento y la fijación de objetivos y la conexión de estos con los intereses del Estado en los ámbitos nacional e internacional; su importancia dentro del proceso de toma de decisiones, etc., todo ello nos conduce también a plantear elementos importantes y novedosos de comparación histórica.

Sabemos que los distintos servicios de inteligencia europeos han crecido, se han desarrollado y se han ido perfeccionando de manera progresiva a lo largo del siglo XX en sintonía con la propia evolución global de los Estados y los paralelos cambios en las relaciones internacionales. El afianzamiento de los sistemas democráticos, no solo no ha sido un impedimento sino que se ha convertido en un acicate más, y cada vez más necesario, para contar con Servicios eficaces, por supuesto, al servicio del Estado. 